\title{
A Further Study on Finding More Minimal Negatively S-Transitive Closures
}

\author{
X.C. Yang, C.P. Wu \\ College of Mathematics, Taiyuan University of Technology \\ Taiyuan, Shanxi, PR China
}

\begin{abstract}
In this paper, we mainly suggested further methods to find more minimal negatively S-transitive closures of a fuzzy relation on a finite universe based on the construction proposed by Han. At the same time, an example was given to illustrate our methods by computer programming. As a result, some minimal negatively S-transitive closures differing from Han are obtained.
\end{abstract}

Keywords--fuzzy relation; negative s-transitivity; minimal negatively s-transitive closure

\section{INTRODUCTION}

In 1965, Zadeh ${ }^{[1]}$ introduced fuzzy binary relations. Afterwards, they are extensively used in a number of fields of decision making analysis $\left.{ }^{[2-5}\right]$. In the study of fuzzy relations, negative S-transitivity property often plays a central role. In 2003, Xue and Wang ${ }^{6]}$ discussed the relationship between T-transitivity and negative S-transitivity. They ${ }^{[7]}$ had a further study on it in 2004. In 2006, Qin and Han ${ }^{[8}$ ] investigated the negatively S-transitive interior of a fuzzy relation.

It is known to all of us that the fuzzy relations are not necessary to satisfy a property $\mathrm{P}$ when the data comes from the practical applications. Bandler and Kohout $\left.{ }^{[9}\right]$ presented the concepts of closure satisfying the property $\mathrm{P}$ for a fuzzy relation and gave a necessary and sufficient condition for the existence of a closure. Han ${ }^{10}$ ] presented firstly a method to find a minimal negatively S-transitive closure in 2012. Based on Han's method, we construct different minimal negatively S-transitive closures from different rows or columns.

The rest of the paper is organized as follows. In Section 2, we present basic definitions and notations. In Section 3, we present our methods to find more minimal negatively S-transitive closures, give mathematical proofs and an example to illustrate the methods. Some concluding remarks in Section 4.

\section{II.PRELIMINARIES}

In this section, we recall some basic notions of fuzzy relations. All the definitions are from ${ }^{[11]}$.

Definition 2.1 A mapping $\mathrm{S}:[0,1] \times[0,1] \rightarrow[0,1]$ is called a $\mathrm{t}$-conorm if it satisfies the symmetry, monotonicity, associativity and boundary condition.

For example, $\mathrm{S}(\mathrm{x}, \mathrm{y})=\min (\mathrm{x}+\mathrm{y}, 1)$ is a $\mathrm{t}$-conorm which is called the Lukasiewicz t-conorm, and denoted by $W^{\prime}$.

Definition 2.2 A mapping $I_{c}:[0,1] \times[0,1] \rightarrow[0,1]$ is called a fuzzy coimplication if $I_{c}(\cdot, y)$ is decreasing, $I_{c}(x, \cdot)$ is increasing, and $I_{c}(1,0)=1, I_{c}(0,0)=I_{c}(1,1)=0$.
For a t-conorm $S$, let $I_{c}(x, y)=\inf \{z \mid S(x, z) \geq y\}$. Then

$I_{c}$ is a fuzzy coimplication, which is called the R-coimplication induced by t-conorm. For example, $I_{c}(x, y)=\max (y-x, 0)$, which is called the Lukasiewicz coimplication.

Definition 2.3 Let $\mathrm{S}$ be a t-conorm. A fuzzy relation on $\mathrm{A}$ is a mapping $R: A \times A \rightarrow[0,1]$. A fuzzy relation $\mathrm{R}$ on $\mathrm{A}$ is called negatively S-transitive if $R(a, b) \leq S(R(a, c), R(c, b))$ for all $a, b, c \in A$.

Definition 2.4 Let $\mathrm{S}$ be a t-conorm and $\mathrm{R}$ be a fuzzy relation on A. The fuzzy relation $\widetilde{R}$ on A is called a minimal negatively S-transitive closure of $\mathrm{R}$ if the following hold:

(1) $\tilde{R}$ is negatively S-transitive;

(2) $R \subseteq \tilde{R}$;

(3) for any negatively S-transitive relation $S$ such that $R \subseteq S \subseteq \widetilde{R}, S=\widetilde{R}$

\section{FIND MORE MINIMAL NEGATIVELY S-TRANSITIVE CLOSURES}

In this section, we first state the construction of a minimal negatively $S$-transitive closure. Suppose that $A=\left\{a_{1}, a_{2}, \cdots, a_{n}\right\}$. We will define $\tilde{R}\left(a_{1}, a\right)$ first for all $a \in A$, then $\widetilde{R}\left(a_{2}, a\right)$ for all $a \in A, \cdots$, finally $\widetilde{R}\left(a_{n}, a\right)$ for all $a \in A$. Therefore, let

$$
\tilde{R}\left(a_{1}, a\right)=R\left(a_{1}, a\right) \quad \forall a \in A .
$$

Assuming that $\tilde{R}\left(a_{j}, a\right)$ is defined, let

$$
\tilde{R}\left(a_{j+1}, a_{k}\right)=\left\{\begin{array}{l}
\max \left\{R\left(a_{j+1}, a_{k}\right), U\left(a_{j+1}, a_{k}\right), V\left(a_{j+1}, a_{k}\right)\right\} \\
\max \left\{R\left(a_{j+1}, a_{k}\right), V\left(a_{j+1}, a_{k}\right)\right\}
\end{array}\right.
$$

if $k \leq j$

if $k>j$

where

$$
\begin{aligned}
& U\left(a_{j+1}, a_{k}\right)=\max _{c \in A} I_{c}\left(\tilde{R}\left(a_{k}, c\right), R\left(a_{j+1}, c\right)\right), \\
& V\left(a_{j+1}, a_{k}\right)=\max _{i \leq j} I_{c}\left(\tilde{R}\left(a_{i}, a_{j+1}\right), \tilde{R}\left(a_{i}, a_{k}\right)\right) .
\end{aligned}
$$


Motivated by this idea, we will start from any columns or rows of $R$, and obtain some different minimal negatively $S$-transitive closures of $R$.

A. Minimal negatively S-transitive closure constructed from any row

Fix two positive integers $l$ and $m(1 \leq l, m \leq n)$ arbitrarily. Exchange the $l$-th row and the $m$-th row, then the $l$-th column and the $m$-th column of $R$ accordingly. The resulting fuzzy relation is denoted by $R^{\prime}$. Find the minimal negatively $S$-transitive closure $\tilde{R}^{\prime}$ of $R^{\prime}$ by employing the constructive method proposed by $\operatorname{Han}^{[10]}$. Exchanging the $l$-th row and the $m$-th row, the $l$-th column and the $m$-th column of $\tilde{R}^{\prime}$ accordingly leads to the matrix $\tilde{R}$.

Now we prove that $\tilde{R}$ is a minimal negatively $S$-transitive closure of $R$.

Theorem 3.1.1 If $\mathrm{R}$ is a negatively S-transitive relation, then $R^{\prime}$ is also a negatively $\mathrm{S}$-transitive relation.

Proof. By the negatively S-transitivity of R, we have

$R\left(a_{i}, a_{j}\right) \leq S\left(R\left(a_{i}, a_{k}\right), R\left(a_{k}, a_{j}\right)\right)$ for

all $i, j, k, 1 \leq i, j, k \leq n$.

Since $R^{\prime}$ is constructed by exchanging the row $l$ and $m$, then

Case $1 i, j \neq l, m$.

If $k \neq l, m$, then

$R^{\prime}\left(a_{i}, a_{j}\right)=R\left(a_{i}, a_{j}\right) \leq S\left(R\left(a_{i}, a_{k}\right), R\left(a_{k}, a_{j}\right)\right)=S\left(R^{\prime}\left(a_{i}, a_{k}\right), R^{\prime}\left(a_{k}, a_{j}\right)\right)$.

If $k=l$, then

$R^{\prime}\left(a_{i}, a_{j}\right)=R\left(a_{i}, a_{j}\right) \leq S\left(R\left(a_{i}, a_{m}\right), R\left(a_{m}, a_{j}\right)\right)=S\left(R^{\prime}\left(a_{i}, a_{k}\right), R^{\prime}\left(a_{k}, a_{j}\right)\right)$.

If $k=m$, then

$R^{\prime}\left(a_{i}, a_{j}\right)=R\left(a_{i}, a_{j}\right) \leq S\left(R\left(a_{i}, a_{l}\right), R\left(a_{l}, a_{j}\right)\right)=S\left(R^{\prime}\left(a_{i}, a_{k}\right), R^{\prime}\left(a_{k}, a_{j}\right)\right)$.

Case $2 i \neq l, m$ and $j=l$.

If $k \neq l, m$, then

$R^{\prime}\left(a_{i}, a_{j}\right)=R\left(a_{i}, a_{m}\right) \leq S\left(R\left(a_{i}, a_{k}\right), R\left(a_{k}, a_{m}\right)\right)=S\left(R^{\prime}\left(a_{i}, a_{k}\right), R^{\prime}\left(a_{k}, a_{j}\right)\right)$.

If $k=l$, then

$R^{\prime}\left(a_{i}, a_{j}\right)=R\left(a_{i}, a_{m}\right) \leq S\left(R\left(a_{i}, a_{m}\right), R\left(a_{m}, a_{m}\right)\right)=S\left(R^{\prime}\left(a_{i}, a_{k}\right), R^{\prime}\left(a_{k}, a_{j}\right)\right)$.

If $k=m$, then

$R^{\prime}\left(a_{i}, a_{j}\right)=R\left(a_{i}, a_{m}\right) \leq S\left(R\left(a_{i}, a_{l}\right), R\left(a_{l}, a_{m}\right)\right)=S\left(R^{\prime}\left(a_{i}, a_{k}\right), R^{\prime}\left(a_{k}, a_{j}\right)\right)$.

Case $3 i \neq l, m$ and $j=m$.

If $k \neq l, m$, then

$R^{\prime}\left(a_{i}, a_{j}\right)=R\left(a_{i}, a_{l}\right) \leq S\left(R\left(a_{i}, a_{k}\right), R\left(a_{k}, a_{l}\right)\right)=S\left(R^{\prime}\left(a_{i}, a_{k}\right), R^{\prime}\left(a_{k}, a_{j}\right)\right)$.

If $k=l$, then the column $l$ and $m$ of $R$. Thus, we have

$$
R^{\prime}\left(a_{i}, a_{j}\right)= \begin{cases}R\left(a_{i}, a_{j}\right) & i, j \neq l, m, \\ R\left(a_{i}, a_{m}\right) & i \neq l, m ; j=l, \\ R\left(a_{i}, a_{l}\right) & i \neq l, m ; j=m, \\ R\left(a_{m}, a_{j}\right) & i=l ; j \neq l, m, \\ R\left(a_{l}, a_{j}\right) & i=m ; j \neq l, m, \\ R\left(a_{m}, a_{l}\right) & i=l ; j=m, \\ R\left(a_{l}, a_{m}\right) & i=m ; j=l, \\ R\left(a_{m}, a_{m}\right) & i=l ; j=l, \\ R\left(a_{l}, a_{l}\right) & i=m ; j=m .\end{cases}
$$

In order to show the negatively $S$-transitivity of $R^{\prime}$, the following inequalities need to be verified for all $i, j, k, 1 \leq i, j, k \leq n$,

$$
R^{\prime}\left(a_{i}, a_{j}\right) \leq S\left(R^{\prime}\left(a_{i}, a_{k}\right), R^{\prime}\left(a_{k}, a_{j}\right)\right) .
$$

Consider the following nine cases. 
$R^{\prime}\left(a_{i}, a_{j}\right)=R\left(a_{i}, a_{l}\right) \leq S\left(R\left(a_{i}, a_{m}\right), R\left(a_{m}, a_{l}\right)\right)=S\left(R^{\prime}\left(a_{i}, a_{k}\right), R^{\prime}\left(a_{k}, a_{j}\right)\right)$.

If $k=m$, then

$R^{\prime}\left(a_{i}, a_{j}\right)=R\left(a_{i}, a_{l}\right) \leq S\left(R\left(a_{i}, a_{l}\right), R\left(a_{l}, a_{l}\right)\right)=S\left(R^{\prime}\left(a_{i}, a_{k}\right), R^{\prime}\left(a_{k}, a_{j}\right)\right)$.

Case $4 i=l$ and $j \neq l, m$.

If $k \neq l, m$, then

$R^{\prime}\left(a_{i}, a_{j}\right)=R\left(a_{m}, a_{j}\right) \leq S\left(R\left(a_{m}, a_{k}\right), R\left(a_{k}, a_{j}\right)\right)=S\left(R^{\prime}\left(a_{i}, a_{k}\right), R^{\prime}\left(a_{k}, a_{j}\right)\right)$.

If $k=l$, then

$R^{\prime}\left(a_{i}, a_{j}\right)=R\left(a_{m}, a_{j}\right) \leq S\left(R\left(a_{m}, a_{m}\right), R\left(a_{m}, a_{j}\right)\right)=S\left(R^{\prime}\left(a_{i}, a_{k}\right), R^{\prime}\left(a_{k}, a_{j}\right)\right)$.

If $k=m$, then

$R^{\prime}\left(a_{i}, a_{j}\right)=R\left(a_{m}, a_{j}\right) \leq S\left(R\left(a_{m}, a_{l}\right), R\left(a_{l}, a_{j}\right)\right)=S\left(R^{\prime}\left(a_{i}, a_{k}\right), R^{\prime}\left(a_{k}, a_{j}\right)\right)$.

Case $5 i=m$ and $j \neq l, m$.

If $k \neq l, m$, then

$R^{\prime}\left(a_{i}, a_{j}\right)=R\left(a_{l}, a_{j}\right) \leq S\left(R\left(a_{l}, a_{k}\right), R\left(a_{k}, a_{j}\right)\right)=S\left(R^{\prime}\left(a_{i}, a_{k}\right), R^{\prime}\left(a_{k}, a_{j}\right)\right)$.

If $k=l$, then

$R^{\prime}\left(a_{i}, a_{j}\right)=R\left(a_{l}, a_{j}\right) \leq S\left(R\left(a_{l}, a_{m}\right), R\left(a_{m}, a_{j}\right)\right)=S\left(R^{\prime}\left(a_{i}, a_{k}\right), R^{\prime}\left(a_{k}, a_{j}\right)\right)$.

If $k=m$, then

$R^{\prime}\left(a_{i}, a_{j}\right)=R\left(a_{l}, a_{j}\right) \leq S\left(R\left(a_{l}, a_{l}\right), R\left(a_{l}, a_{j}\right)\right)=S\left(R^{\prime}\left(a_{i}, a_{k}\right), R^{\prime}\left(a_{k}, a_{j}\right)\right)$.

Case $6 i=l$ and $j=m$.

If $k \neq l, m$, then

$R^{\prime}\left(a_{i}, a_{j}\right)=R\left(a_{m}, a_{l}\right) \leq S\left(R\left(a_{m}, a_{k}\right), R\left(a_{k}, a_{l}\right)\right)=S\left(R^{\prime}\left(a_{i}, a_{k}\right), R^{\prime}\left(a_{k}, a_{j}\right)\right)$.

If $k=l$, then

$R^{\prime}\left(a_{i}, a_{j}\right)=R\left(a_{m}, a_{l}\right) \leq S\left(R\left(a_{m}, a_{m}\right), R\left(a_{m}, a_{l}\right)\right)=S\left(R^{\prime}\left(a_{i}, a_{k}\right), R^{\prime}\left(a_{k}, a_{j}\right)\right)$.

If $k=m$, then

$R^{\prime}\left(a_{i}, a_{j}\right)=R\left(a_{m}, a_{l}\right) \leq S\left(R\left(a_{m}, a_{l}\right), R\left(a_{l}, a_{l}\right)\right)=S\left(R^{\prime}\left(a_{i}, a_{k}\right), R^{\prime}\left(a_{k}, a_{j}\right)\right)$.

Case $7 i=m$ and $j=l$.

If $k \neq l, m$, then

$R^{\prime}\left(a_{i}, a_{j}\right)=R\left(a_{l}, a_{m}\right) \leq S\left(R\left(a_{l}, a_{k}\right), R\left(a_{k}, a_{m}\right)\right)=S\left(R^{\prime}\left(a_{i}, a_{k}\right), R^{\prime}\left(a_{k}, a_{j}\right)\right)$.

If $k=l$, then

$R^{\prime}\left(a_{i}, a_{j}\right)=R\left(a_{l}, a_{m}\right) \leq S\left(R\left(a_{l}, a_{m}\right), R\left(a_{m}, a_{m}\right)\right)=S\left(R^{\prime}\left(a_{i}, a_{k}\right), R^{\prime}\left(a_{k}, a_{j}\right)\right)$.

If $k=m$, then

$R^{\prime}\left(a_{i}, a_{j}\right)=R\left(a_{l}, a_{m}\right) \leq S\left(R\left(a_{l}, a_{l}\right), R\left(a_{l}, a_{m}\right)\right)=S\left(R^{\prime}\left(a_{i}, a_{k}\right), R^{\prime}\left(a_{k}, a_{j}\right)\right)$.

Case $8 i=j=l$. 
If $k \neq l, m$, then

$R^{\prime}\left(a_{i}, a_{j}\right)=R\left(a_{m}, a_{m}\right) \leq S\left(R\left(a_{m}, a_{k}\right), R\left(a_{k}, a_{m}\right)\right)=S\left(R^{\prime}\left(a_{i}, a_{k}\right), R^{\prime}\left(a_{k}, a_{j}\right)\right)$.

If $k=l$, then

$R^{\prime}\left(a_{i}, a_{j}\right)=R\left(a_{m}, a_{m}\right) \leq S\left(R\left(a_{m}, a_{m}\right), R\left(a_{m}, a_{m}\right)\right)=S\left(R^{\prime}\left(a_{i}, a_{k}\right), R^{\prime}\left(a_{k}, a_{j}\right)\right)$.

If $k=m$, then

$R^{\prime}\left(a_{i}, a_{j}\right)=R\left(a_{m}, a_{m}\right) \leq S\left(R\left(a_{m}, a_{l}\right), R\left(a_{l}, a_{m}\right)\right)=S\left(R^{\prime}\left(a_{i}, a_{k}\right), R^{\prime}\left(a_{k}, a_{j}\right)\right)$.

Case $9 i=j=m$.

If $k \neq l, m$, then

$R^{\prime}\left(a_{i}, a_{j}\right)=R\left(a_{l}, a_{l}\right) \leq S\left(R\left(a_{l}, a_{k}\right), R\left(a_{k}, a_{l}\right)\right)=S\left(R^{\prime}\left(a_{i}, a_{k}\right), R^{\prime}\left(a_{k}, a_{j}\right)\right)$.

If $k=l$, then

$R^{\prime}\left(a_{i}, a_{j}\right)=R\left(a_{l}, a_{l}\right) \leq S\left(R\left(a_{l}, a_{m}\right), R\left(a_{m}, a_{l}\right)\right)=S\left(R^{\prime}\left(a_{i}, a_{k}\right), R^{\prime}\left(a_{k}, a_{j}\right)\right)$.

If $k=m$, then

$R^{\prime}\left(a_{i}, a_{j}\right)=R\left(a_{l}, a_{l}\right) \leq S\left(R\left(a_{l}, a_{l}\right), R\left(a_{l}, a_{l}\right)\right)=S\left(R^{\prime}\left(a_{i}, a_{k}\right), R^{\prime}\left(a_{k}, a_{j}\right)\right)$.

We prove (I) in all cases. Therefore, $R^{\prime}$ is negatively S-transitive.

Theorem 3.1.2 The matrix $\widetilde{R}$ obtained by the previous procedure is a minimal negatively S-transitive closure of $\mathrm{R}$.

Proof. First, we prove that $R \subseteq \tilde{R}$.

By the definition of $\widetilde{R}$ and $\widetilde{R}^{\prime}$, we consider the following cases.

(1) If $i, j \neq l$ and $i, j \neq m$, then

$R\left(a_{i}, a_{j}\right)=R^{\prime}\left(a_{i}, a_{j}\right) \leq \tilde{R^{\prime}}\left(a_{i}, a_{j}\right)=\tilde{R}\left(a_{i}, a_{j}\right)$.

(2) If $i \neq l, m$ and $j=l$, then $R\left(a_{i}, a_{j}\right)=R^{\prime}\left(a_{i}, a_{m}\right) \leq \tilde{R^{\prime}}\left(a_{i}, a_{m}\right)=\tilde{R}\left(a_{i}, a_{j}\right)$.

(3) If $i \neq l, m$ and $j=m$, then $R\left(a_{i}, a_{j}\right)=R^{\prime}\left(a_{i}, a_{l}\right) \leq \tilde{R^{\prime}}\left(a_{i}, a_{l}\right)=\tilde{R}\left(a_{i}, a_{j}\right)$.

(4) If $i=l$ and $j \neq l, m$, then

$R\left(a_{i}, a_{j}\right)=R^{\prime}\left(a_{m}, a_{j}\right) \leq \tilde{R^{\prime}}\left(a_{m}, a_{j}\right)=\tilde{R}\left(a_{i}, a_{j}\right)$.

(5) If $i=l$ and $j \neq l, m$, then $R\left(a_{i}, a_{j}\right)=R^{\prime}\left(a_{l}, a_{j}\right) \leq \tilde{R^{\prime}}\left(a_{l}, a_{j}\right)=\tilde{R}\left(a_{i}, a_{j}\right)$.

(6) If $i=l$ and $j=m$, then $R\left(a_{i}, a_{j}\right)=R^{\prime}\left(a_{m}, a_{l}\right) \leq \tilde{R^{\prime}}\left(a_{m}, a_{l}\right)=\tilde{R}\left(a_{i}, a_{j}\right)$.

(7) If $i=m$ and $j=l$, then $R\left(a_{i}, a_{j}\right)=R^{\prime}\left(a_{l}, a_{m}\right) \leq \tilde{R^{\prime}}\left(a_{l}, a_{m}\right)=\tilde{R}\left(a_{i}, a_{j}\right)$.

(8) If $i=j=l$, then $R\left(a_{i}, a_{j}\right)=R^{\prime}\left(a_{m}, a_{m}\right) \leq \tilde{R^{\prime}}\left(a_{m}, a_{m}\right)=\tilde{R}\left(a_{i}, a_{j}\right)$.

(9) If $i=j=m$, then $R\left(a_{i}, a_{j}\right)=R^{\prime}\left(a_{l}, a_{l}\right) \leq \tilde{R^{\prime}}\left(a_{l}, a_{l}\right)=\tilde{R}\left(a_{i}, a_{j}\right)$. 
Thus, $R \subseteq \tilde{R}$ is verified in all cases.

Next, we show that $\tilde{R}$ is negatively S-transitive.

Since $\widetilde{R}^{\prime}$ is a minimal negatively $\mathrm{S}$-transitive closure of $R^{\prime}, \widetilde{R}^{\prime}$ is negatively S-transitive. Observing that $\widetilde{R}$ is obtained by exchanging the row $\mathrm{l}$ and $\mathrm{m}$, the column $\mathrm{l}$ and $\mathrm{m}$ of $\widetilde{R}^{\prime}$, it is negatively S-transitive by Theorem 3.1.1.

Finally, we prove that $\tilde{R}$ is minimal. Suppose that there is a negatively S-transitive fuzzy relation $\mathrm{S}$ such that $R \subseteq S \subseteq \tilde{R}$. Exchange the row $\mathrm{l}$ and $\mathrm{m}$ and the column $\mathrm{l}$ and $\mathrm{m}$ of $R, S, \widetilde{R}$ to obtain matrices $R^{\prime}, S^{\prime}, \tilde{R}^{\prime} \quad$ respectively. Clearly, $R^{\prime} \subseteq S^{\prime} \subseteq \widetilde{R}^{\prime}$. By Theorem 3.1.1, $S^{\prime}$ is negatively S-transitive. Since $\widetilde{R}^{\prime}$ is a minimal negatively $\mathrm{S}$-transitive closure of $R^{\prime}$, we have $\widetilde{R}^{\prime}=S^{\prime}$. Therefore, $\tilde{R}=S$. of R.

In a word, $\tilde{R}$ is a minimal negatively S-transitive closure

\section{B. Minimal negatively S-transitive closure constructed from any column}

In the sequel, we denote the transposition of a fuzzy relation $S$ by $S^{T}$.

Theorem 3.2.1 If $\mathrm{R}$ is a negatively $\mathrm{S}$-transitive relation, then $R^{T}$ is also negatively $\mathrm{S}$-transitive.

Proof. By the negatively S-transitivity of R,

$$
R\left(a_{i}, a_{j}\right) \leq S\left(R\left(a_{i}, a_{k}\right), R\left(a_{k}, a_{j}\right)\right) \quad i, j, k=1,2, \cdots, n .
$$

For all $i, j, k$,

$$
\begin{aligned}
& \quad S\left(R^{T}\left(a_{i}, a_{k}\right), R^{T}\left(a_{k}, a_{j}\right)\right)=S\left(R\left(a_{k}, a_{i}\right), R\left(a_{j}, a_{k}\right)\right)=S\left(R\left(a_{j}, a_{k}\right), R\left(a_{k}, a_{i}\right)\right) \\
& \geq R\left(a_{j}, a_{i}\right)=R^{T}\left(a_{i}, a_{j}\right) .
\end{aligned}
$$

Therefore, $R^{T}$ is negatively $S$-transitive.

Theorem 3.2.2 If $R^{T}$ is a minimal negatively S-transitive closure of $R^{T}$, then $\widetilde{R}=\left(R^{T}\right)^{T}$ is a minimal negatively S-transitive closure of $\mathrm{R}$.

Proof. Since $\widetilde{R^{T}}$ is a minimal negatively $S$-transitive closure of $R^{T}$, we have $R^{T} \subseteq R^{T}$, and hence $R \subseteq \widetilde{R}$. It follows from the negatively $S$-transitivity of $R^{T}$ and Theorem 3.2.1 that $\tilde{R}=\left(R^{T}\right)^{T}$ is also negatively $S$-transitive.

To show that $\widetilde{R}$ is minimal, suppose that there is a negatively $S$-transitive fuzzy relation $S$ such that $R \subseteq S \subseteq \tilde{R}$. Then, $R^{T} \subseteq S^{T} \subseteq \widetilde{R}^{T}$. By Theorem 3.2.1, $S^{T}$ is also negatively $S$-transitive. Since $\widetilde{R^{T}}$ is a minimal negatively
$S$-transitive closure of $R^{T}, \quad R^{T}=S^{T}$, i. e. $\tilde{R}=S$. In summary, $\widetilde{R}$ is a minimal negatively $S$-transitive closure of $R$.

Based on the previous theorems, we present a procedure to find minimal negatively $S$-transitive closures starting from a column. Exchange the row $l$ and $m$, the column $l$ and $m$ of $R$ to obtain $R_{1}$. Find the minimal negatively $S$-transitive closure $\widetilde{R_{1}^{T}}$ of $R_{1}^{T}$ by the method proposed by Han. Let $\widetilde{R}_{1}=\left(\widetilde{R_{1}^{T}}\right)^{T}$. Obtain $\tilde{R}$ by exchanging the row $l$ and $m$, the column $l$ and $m$ of $\widetilde{R}_{1}$. Then, $\tilde{R}$ is a minimal negatively $S$-transitive closure of $R$ by Theorem 3.1.2, Theorem 3.2.1 and Theorem 3.2.2.

\section{An example}

Let $S(x, y)=W^{\prime}(x, y)=\min (x+y, 1)$,

and $I_{W^{\prime}}(x, y)=\max (y-x, 0) . A=\{a, b, c\}$ and define $R$ on A by

$$
R=\left(\begin{array}{ccc}
0.9 & 0.1 & 0.3 \\
0.4 & 0.8 & 0 \\
0.1 & 0.1 & 0.7
\end{array}\right)
$$

Clearly, $R$ is not negatively $S$-transitive. Now we find minimal negatively $S$-transitive closures of $R$ by the proposed methods.

Method 1. Find minimal negatively $S$-transitive closures starting from rows. The obtained minimal negatively $S$-transitive closures are respectively

$$
\begin{aligned}
\widetilde{R}_{1}=\left(\begin{array}{ccc}
0.9 & 0.1 & 0.3 \\
0.8 & 0.8 & 0.2 \\
0.6 & 0.6 & 0.7
\end{array}\right) & , & \widetilde{R}_{2}=\left(\begin{array}{lll}
0.9 & 0.1 & 0.3 \\
0.8 & 0.8 & 0.7 \\
0.6 & 0.1 & 0.7
\end{array}\right), \\
\widetilde{R}_{3}=\left(\begin{array}{ccc}
0.9 & 0.5 & 0.3 \\
0.4 & 0.8 & 0 \\
0.6 & 0.8 & 0.7
\end{array}\right) & , & \widetilde{R}_{4}=\left(\begin{array}{ccc}
0.9 & 0.5 & 0.5 \\
0.4 & 0.8 & 0 \\
0.4 & 0.8 & 0.7
\end{array}\right), \\
\widetilde{R}_{5}=\left(\begin{array}{ccc}
0.9 & 0.1 & 0.8 \\
0.8 & 0.8 & 0.7 \\
0.1 & 0.1 & 0.7
\end{array}\right) & , & \widetilde{R}_{6}=\left(\begin{array}{ccc}
0.9 & 0.5 & 0.8 \\
0.4 & 0.8 & 0.7 \\
0.1 & 0.1 & 0.7
\end{array}\right) .
\end{aligned}
$$

Method 2. Find minimal negatively $S$-transitive closures starting from columns. The obtained minimal negatively $S$-transitive closures differing from Method 1 are:

$$
\widetilde{R}_{7}=\left(\begin{array}{ccc}
0.9 & 0.5 & 0.8 \\
0.4 & 0.8 & 0.3 \\
0.1 & 0.5 & 0.7
\end{array}\right) \quad, \quad \widetilde{R}_{8}=\left(\begin{array}{ccc}
0.9 & 0.3 & 0.3 \\
0.6 & 0.8 & 0 \\
0.6 & 0.8 & 0.7
\end{array}\right) .
$$

Therefore, we find eight different minimal negatively $S$-transitive closures among which $\widetilde{R}_{1}$ is obtained in [10]. 


\section{CONCLUDING REMARKS}

In this paper, we give several methods to find more minimal negatively S-transitive closures of a fuzzy relation. Then, we make a comparison with the results in [10] by an example. In view of the complication of calculating the minimal negatively S-transitive closure, especially when $n$ is larger, we realize it on computer by programming.

\section{ACKNOWLEDGMENTS}

This research is financially supported by the Science and Technology Innovation Foundation of Taiyuan University of Technology for Graduate Students, Natural Science Foundation of Shanxi Province (NO. 2013011004-1) and Shanxi Scholarship Council of China (NO. 2013052).

\section{REFERENCES}

[1] Zadeh, L.A., Fuzzy sets, Information and Control, 8, pp. 338-353, 1965.

[2] Wang, X.Z., Ruan, D., Kerre, E., Mathematics of fuzziness-basic issues, Springer-Verlag:Berlin,2007.

[3] Wang, X.Z., Kerre, E., Reasonable properties for the ordering of fuzzy quantities( II ), Fuzzy Sets and System, 118, pp. 387-405, 2001

[4] Georgescu, I., Fuzzy choice functions, Springer-Verlag: Berlin, 2007.

[5] Díaz, S., De Baets, B., Montes, S., On the Ferrers property of valued interval orders, Top 19 (2), pp. 421-427, 2011.

[6] Xue, Y., Wang, X.Z, On the relationship between T-transitivity and S-negative transitivity, Fuzzy Systems and Mathematics, 17(3), pp. 72-76, 2003.

[7] Wang, X.Z., Xue, Y., Note on transitivity, negative transitivity, semitransitivity and Ferrers property, The Journal of Fuzzy Mathematics, 12, pp. 323-330, 2004.

[8] Qin, X.Y., Han, H.J., Research on the negative S-transitive interior of a fuzzy relation, Journal of Taiyuan University of Technology, 37(4), pp. 489-491, 2006.

[9] Bandler, W., Kohout, L.J., Special properties, closures and interiors of crisp and fuzzy relations,Fuzzy Sets and System, 26, pp. 317-331, 1988.

[10] Han, H.J., One method of finding a minimal negative S-transitive closure, Journal of Taiyuan University of Technology, 43(4), pp. 527-530, 2012.

[11] Fodor, J.C., Roubens, M., Fuzzy preference modelling and multicriteria decision support KluwerAcademic Publishers: Dordercht/Boston/London, 1994. 